Vietnam Journal of Mechanics, VAST, Vol.40, No. 4 (2018), pp. $397-406$

DOI: https://doi.org/10.15625/0866-7136/13215

\title{
INFLUENCE OF THE DRIVING FREQUENCY AND EQUIVALENT PARAMETERS ON DISPLACEMENT AMPLITUDE OF ELECTROSTATIC LINEAR COMB ACTUATOR
}

\author{
Hoang Trung Kien ${ }^{1}$, Vu Cong Ham ${ }^{2}$, Pham Hong Phuc ${ }^{1, *}$ \\ ${ }^{1}$ Hanoi University of Science and Technology, Vietnam \\ ${ }^{2}$ Le Quy Don University, Vietnam \\ *E-mail: phuc.phamhong@hust.edu.vn \\ Received Octorber 22, 2018
}

\begin{abstract}
A new method determining the equivalent dynamic parameters such as stiffness, vibrating mass, and air damping factor in motion direction of shuttle (i.e. in $y$ direction) is proposed, thence the differential motion equation of shuttle is established and solved to achieve a typical displacement formula. Simulation and experimental results show that the change of ELCA' displacement is inappreciable while the range of driving frequency up to $27 \mathrm{~Hz}$ (error of $10 \%$ with driving voltage is a square wave). Moreover, the range of driving frequency for the ELCA can be extended up to $1 \mathrm{kHz}$ with displacement amplitude error of $10 \%$ while the shape of driving voltage is a harmonic sine wave.
\end{abstract}

Keywords: Electrostatic Linear Comb Actuator (ELCA), driving frequency, dynamic parameters, displacement amplitude.

\section{INTRODUCTION}

Micro electromechanical system (MEMS) is an advance technology and widely applied in many fields like robotics, transportation, aerospace, medicine, or in electronic industry, etc. Micro actuators are one of the MEMS products and play an important role which generates a power to drive the micro devices as well as micro systems. Electrostatic Linear Comb Actuator (ELCA) is a typical actuator with working principle based on the electrostatic force producing linear movement. The advantages of the ELCA system are fast response, high performance, low energy consumption, and simple configuration. That is the reason why it is commonly used for driving MEMS devices, such as resonators [1], gyroscopes [2], micro conveyer/transportation systems [3], micro motor [4], or micro gripper [5].

(C) 2018 Vietnam Academy of Science and Technology 
Recently, there are many researches on the ELCA in various aspects. They cover main topics such as: new structures, working quality, manufacturing process improvement, and application of smart materials. Particularly, the fabrication quality as well as the stability of the ELCA's motion determines the working accuracy of the devices. The shapes of suspension beams such as a crab-leg and fold $[6,7]$ have been designed in order to reduce the stiffness and improve the displacement. Another research on improving displacement has been published [8], by reducing out-of-plane movement from that establishes in-plane displacement. The authors in [9] have proposed a structure of an actuator by adding a module which combines of a sensor and a sub-actuator for controlling and minimizing instability. The influence of side etching effect on displacement of the ELCA after deep-Reactive ion etching (D-RIE) process has been mentioned particularly by [10].

When calculating the displacement of the ELCA in design task [3,4,10-12], the authors used the theoretical formula which is established based on static equilibrium equation, aiming simplifies computing and reduces a design time. In fact, these devices work in state of motion though applying $\mathrm{AC}$ voltage likes a square or sine wave, etc. Therefore, considering the influence of inertia force and air damping plays an important role in predicting the fact displacement of the ELCA. The dynamic of the ELCA has studied in $[13,14]$, in which the electrostatic actuators are used for driving resonators and gyroscopes. However, the parameters of differential equation of motion such as an effective mass and conversion air damping factor have not clearly determined in these publications.

In this paper, we propose equivalent dynamic parameters, i.e. the effective mass of the ELCA's movable part and conversion air damping factor via equivalent transformation kinetic energy and the air drag method. The ELCA's displacement is examined and discussed corresponding to the change of driving frequency by solving the differential equation of motion.

\section{THEORETICAL DISPLACEMENT OF ELCA}

\subsection{Configuration and displacement of ELCA}

Fig. 1 shows the configuration of ELCA, which consists of a movable part (shuttle) (1) and fixed part (anchor pads (2) and fixed electrodes (3)). The movable part (consists of movable comb fingers) which is suspended by four elastic beams system moving in $y$-direction under the electrostatic force, one end of each beam is fixed on anchor pad (2). The structure and dimensions of movable and fixed comb fingers are the same. Tab. 1 describes the geometric parameters of ELCA as shown in Fig. 1, where $b$ is the width of a beam, $h$ is the thickness of device layer, and $g_{a}$ is the gap between the shuttle's bottom and substrate.

Table 1. Value of the ELCA geometric parameters

\begin{tabular}{ccccc}
\hline & Length $(\mu \mathrm{m})$ & Width $(\mu \mathrm{m})$ & Thickness $(\mu \mathrm{m})$ & Gap $(\mu \mathrm{m})$ \\
\hline Comb finger & $l_{c}=40$ & $w=3$ & $h=30$ & $g=2$ \\
Suspension beam & $L=515$ & $b=3.6$ & $h=30$ & $g_{a}=4$ \\
\hline
\end{tabular}




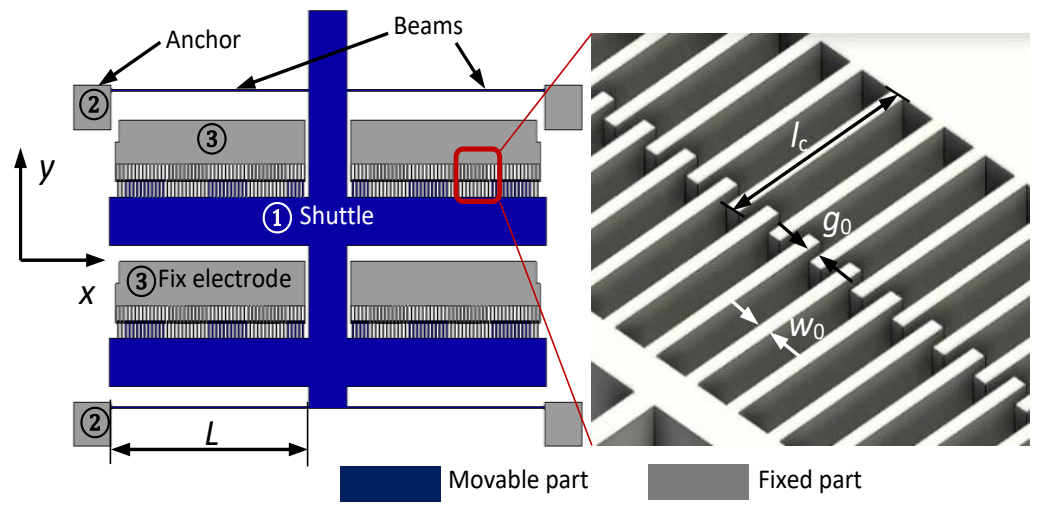

Fig. 1. Configuration of ELCA

While applying a voltage $V$ between anchor pads (2) and fixed electrodes (3) of the ELCA, the total electrostatic force in $y$-direction is expressed as follows

$$
F_{e}=\frac{n h \varepsilon \varepsilon_{0}}{g_{0}} V^{2}
$$

where $n$ is a total number of the movable comb fingers; $\varepsilon_{0}=8.854 \times 10^{-6} \mathrm{pF} / \mu \mathrm{m}$ and $\varepsilon=1$ are permittivity of vacuum and air, respectively; $V$ is the driving voltage.

The total stiffness in $y$-direction of four suspension beams system can be calculated as $[4,10]$

$$
K=\frac{4 E h b^{3}}{L^{3}}
$$

where $E=169 \mathrm{GPa}$ is silicon Young's modulus.

While applying a $D C$ voltage for ELCA, the displacement $y_{0}$ of ELCA (i.e. displacement of the shuttle) is directly inferred from force balance equation

$$
F_{e}-F_{e l}=0 \text { or } \frac{n h \varepsilon \varepsilon_{0}}{g_{0}} V^{2}=\frac{4 E h b^{3}}{L^{3}} y_{0} .
$$

Here, $F_{e l}$ is a total elastic force of suspension beams. We have

$$
y_{0}=\frac{n \varepsilon \varepsilon_{0}}{4 E g_{0}}\left(\frac{L}{b}\right)^{3} V^{2}
$$

Eq. (3) indicates the quadratic relation between the displacement of the ELCA and the $D C$ driving voltage.

\subsection{Dynamic model of ELCA}

In case driving voltage is an AC: considering the motion of the ELCA's shuttle, this motion is similar to the motion of a mass with one-degree of freedom shown in Fig. 2. $K, C, M$ are the conversion stiffness, conversion damping coefficient and conversion effective mass of the ELCA system, respectively. $F_{e}(t)$ is an external total force (here is an electrostatic driving force). 


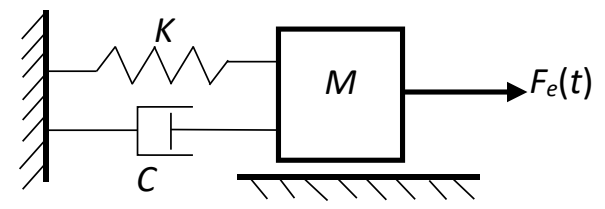

Fig. 2. 1-DOF dynamic model of the ELCA

The differential equation of ELCA's motion in y-direction (Fig. 1) can be expressed

$$
M \ddot{y}+C \dot{y}+K y=F_{e}(t) .
$$

In order to calculate exactly the displacement of the ELCA through differential equation, the equivalent coefficients $K, M, C$ must be accurately identified. These coefficients depend on various parameters such as geometric dimensions, material properties, working environment, etc. of the system.

The conversion stiffness $K$ in $y$-direction can be determined by Eq. (2) above.

The effective mass of movable parts (including the elastic beams) converted to shuttle's motion is based on rule: the kinetic energy of effective mass $M$ is equal to total kinetic energy of all movable parts of the ELCA, thus we have

$$
M=M_{s}+\frac{4 M_{b}}{L} \int_{0}^{L}\left(\frac{\dot{y}(x)}{\dot{y}(L)}\right)^{2} \mathrm{~d} x,
$$

where $M_{s}, M_{b}$ are the mass of the shuttle and of one single beam, respectively; $\dot{y}(L), \dot{y}(x)$ are the velocity of the shuttle and the section of a single beam at $x$-position.

We assume that directional movement of the shuttle and beam cross-sections at $x$ position are the same. Thus, relation between $\dot{y}(x)$ and $\dot{y}(L)$ is obtained through the relation of displacements

$$
y(x)=\frac{3 L x^{2}-2 x^{3}}{L^{3}} y(L) \Rightarrow \dot{y}(x)=\frac{3 L x^{2}-2 x^{3}}{L^{3}} \dot{y}(L) .
$$

From (5) and (6), we have

$$
M=M_{s}+4 \frac{13 M_{b}}{35}=\rho h\left(A_{t}+\frac{52 b L}{35}\right),
$$

where $\rho=2330 \mathrm{~kg} / \mathrm{m}^{3}$ is the mass density of silicon; $A_{t}$ is the total top side area of the shuttle.

According to [15], the conversion air-damping coefficient $C$ in Eq. (4) can be calculated

$$
C=C_{1}+C_{2}+C_{3}
$$

$C_{1}$ is the air slide-film damping coefficient on shuttle's bottom and movable fingers' face versus substrate and fixed fingers, respectively

$$
C_{1}=\mu\left(\frac{A_{b t}}{g_{a}}+\frac{2 A_{s c}}{g_{0}}\right)
$$


where $\mu=1.85 \times 10^{-5} \mathrm{~Pa} \cdot \mathrm{s}$ (at room temperature $T_{0}=20^{\circ} \mathrm{C}$ ) is the viscosity coefficient of air; $A_{b t}, A_{s c}$ are the total areas of the shuttle's bottom and comb fingers' face, respectively.

$C_{2}$ is the air slide-film damping coefficient on the top and two sides of the shuttle

$$
C_{2}=\mu \frac{A_{t}+A_{s s}}{\delta}
$$

where $A_{t}, A_{s s}$ are the top and two sides area of the shuttle; $\delta=\sqrt{\frac{2 \mu}{\rho_{a} \omega}}$ is the effective distance; $\rho_{a}$ is the mass density of air, $\omega$ is the angle frequency of system. $C_{2}$ is trivial and can be ignored in some cases.

$C_{3}$ is the air drag coefficient on the vertical surface of the movable part and can be approximated as follows

$$
C_{3} \approx \frac{32}{3} \mu \frac{h}{2}
$$

Using Eqs. (8), (9) and (10), we have

$$
C=\mu\left(\frac{A_{b t}}{g_{a}}+\frac{2 A_{s c}}{g_{0}}+\frac{A_{t}+A_{s s}}{\delta}+\frac{16 h}{3}\right) .
$$

The force $F_{e}(t)$ in Eq. (4) is a total electrostatic force, which is generated by applying voltage on two electrodes of the ELCA. This force depends on amplitude, frequency and kind of driving voltage wave.

In case of the driving voltage is the square wave as in Fig. 3.

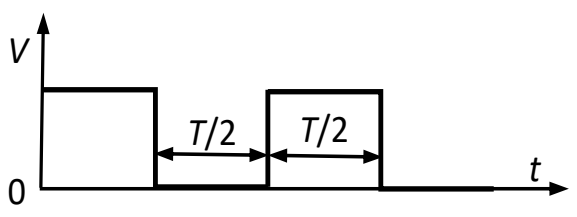

Fig. 3. Square wave of driving voltage

The $F_{e}(t)$ force function can be expressed as

$$
F_{e}(t)=\left\{\begin{array}{cc}
\frac{n h \varepsilon \varepsilon_{0}}{g_{0}} V^{2}, & 0 \leq t \leq T / 2 \\
0, & T / 2<t \leq T
\end{array}\right.
$$

where $T$ is one cycle of the applying voltage. Accordingly, the displacement of differential equation (4) has the form

$$
y_{1}=e^{-\beta t}\left(A_{1} \cos (\omega t)+A_{2} \sin (\omega t)\right)+\frac{F_{e}}{K} .
$$

Here $\beta=\frac{C}{2 M} ; \omega=\sqrt{\omega_{n}^{2}-\beta^{2}}$ is the damped angle frequency; $\omega_{n}=\sqrt{\frac{K}{M}}$ is the natural angle frequency. 
According to the initial condition: $y(0)=0$ and $\dot{y}(0)=0$, we have: $A_{1}=-\frac{F_{e}}{K}$; $A_{2}=-\frac{\beta}{\omega} \frac{F_{e}}{K}$.

The general displacement formula of the shuttle in this case can be expressed

$$
y_{1}=\frac{n \varepsilon \varepsilon_{0}}{4 E g_{0}}\left(\frac{L}{b}\right)^{3} V^{2}\left[1-\left(\cos (\omega t)+\frac{\beta}{\omega} \sin (\omega t)\right) e^{-\beta t}\right] .
$$

In case of the driving voltage is the sine wave function as shown in Fig. 4.

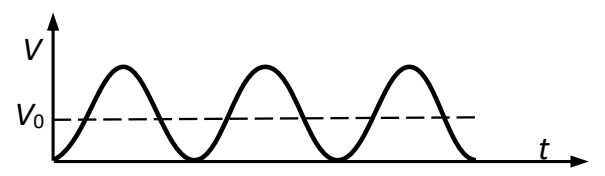

Fig. 4. Sine wave of driving voltage $V=V_{0}(1+\sin (\Omega t))$

By setting: $F_{0}=\frac{n h \varepsilon \varepsilon_{0}}{g_{0}} V_{0}^{2}$, the driving force can be expressed as

$$
F_{e s}=F_{0}\left[\frac{3}{2}+2 \sin (\Omega t)-\frac{1}{2} \cos (2 \Omega t)\right]
$$

In this case, the displacement result of differential equation (4) is calculated

$$
\begin{aligned}
y_{1 s} & =\frac{3 F_{0}}{2 K}+\frac{2 F_{0}}{\left(K-M \Omega^{2}\right)^{2}+C^{2} \Omega^{2}}\left[\left(K-M \Omega^{2}\right) \sin (\Omega t)-C \Omega \cos (\Omega t)\right] \\
& -\frac{F_{0}}{2\left(K-4 M \Omega^{2}\right)^{2}+8 C^{2} \Omega^{2}}\left[\left(K-4 M \Omega^{2}\right) \cos (2 \Omega t)+2 C \Omega \sin (2 \Omega t)\right] \\
& +e^{-\beta t}\left[B_{1} \cos (\omega t)+B_{2} \sin (\omega t)\right] .
\end{aligned}
$$

According to initial condition: $y_{1 s}(0)=0$ and $\dot{y}_{1 s}(0)=0$, we have

$$
\begin{aligned}
& B_{1}=F_{0}\left\{\frac{2 C \Omega}{\left(K-M \Omega^{2}\right)^{2}+C^{2} \Omega^{2}}+\frac{K-4 M \Omega^{2}}{2\left(K-4 M \Omega^{2}\right)^{2}+8 C^{2} \Omega^{2}}-\frac{3}{2 K}\right\}, \\
& B_{2}=\frac{F_{0}}{\omega}\left\{\frac{2 C \Omega^{2}}{\left(K-4 M \Omega^{2}\right)^{2}+4 C^{2} \Omega^{2}}-\frac{2 \Omega\left(K-M \Omega^{2}\right)}{\left(K-M \Omega^{2}\right)^{2}+C^{2} \Omega^{2}}\right\}+\frac{\beta}{\omega} B_{1} .
\end{aligned}
$$

\section{ANALYSIS, SIMULATION AND MEASUREMENT RESULTS}

Material properties of the silicon $[4,10]$ and air are listed in Tab. 2. The conversion coefficients in differential equation (4) calculated by Eqs. (2), (7) and (11) are shown in Tab. 3.

The ELCA's simulation is implemented by using a finite element method (FEM) in ANSYS. At driving voltage of $100 \mathrm{~V}$, the maximum stress on suspension beams is 28.2 $\mathrm{MPa}$ and the obtained displacement $y$ of the shuttle is $38.42 \mu \mathrm{m}$, as shown in Fig. 5 . 
Table 2. Material properties of silicon and air

\begin{tabular}{lll}
\hline Young's modulus of silicon & $E(\mathrm{~Pa})$ & $1.69 \mathrm{E}+11$ \\
\hline Mass density of silicon & $\rho\left(\mathrm{kg} / \mathrm{m}^{3}\right)$ & 2330 \\
Mass density of air $\left(20^{\circ} \mathrm{C}, 1 \mathrm{~atm}\right)$ & $\rho_{a}\left(\mathrm{~kg} / \mathrm{m}^{3}\right)$ & 1.205 \\
Viscosity coefficient of air $\left(20^{\circ} \mathrm{C}, 1 \mathrm{~atm}\right)$ & $\mu(\mathrm{Pa} \cdot \mathrm{s})$ & $1.85 \mathrm{E}-5$ \\
Permittivity constant of vacuum & $\varepsilon(\mathrm{pF} / \mu \mathrm{m})$ & $8.854 \mathrm{E}-6$ \\
Permittivity coefficient of air & $\varepsilon$ & 1 \\
\hline
\end{tabular}

Table 3. Conversion coefficients of the ELCA

\begin{tabular}{lll}
\hline Conversion stiffness & $K(\mu \mathrm{N} / \mu \mathrm{m})$ & 6.927 \\
\hline Conversion effective mass & $M(\mathrm{~kg})$ & $1.008 \mathrm{E}-08$ \\
Conversion damping & $C(\mu \mathrm{N} \cdot \mathrm{s} / \mu \mathrm{m})$ & $2.322 \mathrm{E}-06$ \\
\hline
\end{tabular}

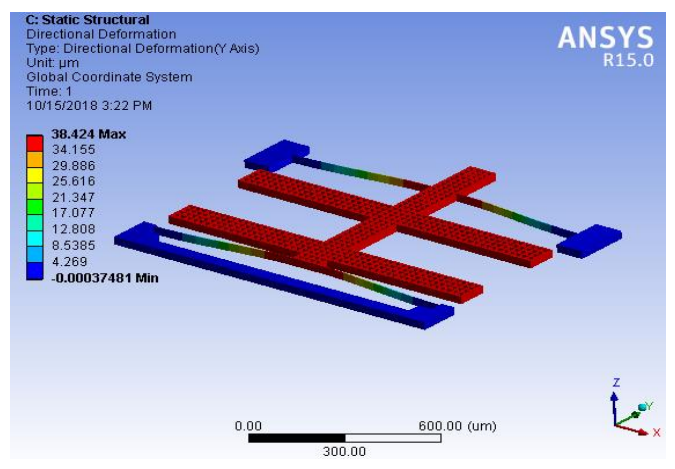

(a) Displacement

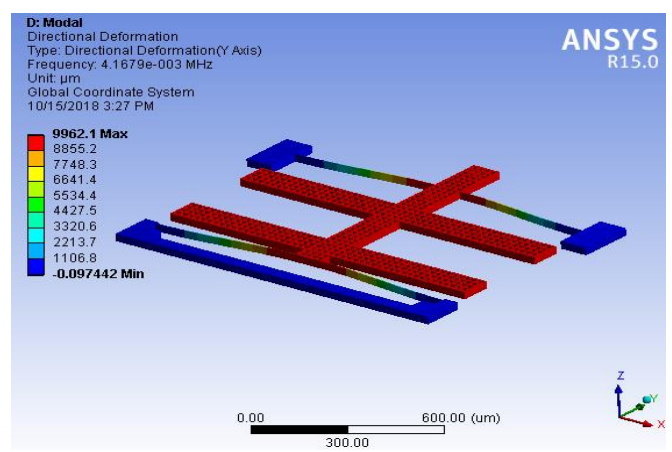

(b) Natural frequency

Fig. 5. Simulation of the ELCA

The natural frequency $\omega_{n}$ of the system determined by theoretical analysis is $4172 \mathrm{~Hz}$, and by ANSYS is $4168 \mathrm{~Hz}$, i.e. deviation is only $0.1 \%$.

The model of ELCA is fabricated by using standard SOI-MEMS technology with a $30 \mu \mathrm{m}$ thick silicon device layer, $4 \mu \mathrm{m}$ thick buried silicon dioxide $\mathrm{SiO}_{2}$ and $450 \mu \mathrm{m}$ thick silicon substrate of SOI (silicon-on-insulator) wafer. Details of fabrication process, such as photolithography, DRIE, and vapor HF etching can be referred in our previous publications [3,4]. Scanning Electron Microscopy (SEM) images of the structures after fabrication is shown in Fig. 6.

Graph on Fig. 7 compares the displacement's value of the shuttle in four cases: theoretical calculation via Eq. (3); influence of conversion coefficients $K, M, C$ via Eq. (14); simulation at $f=1 \mathrm{~Hz}$ and experimental results referred in [10]. The displacement results calculated by theoretical are matching to the results when considering influence of 


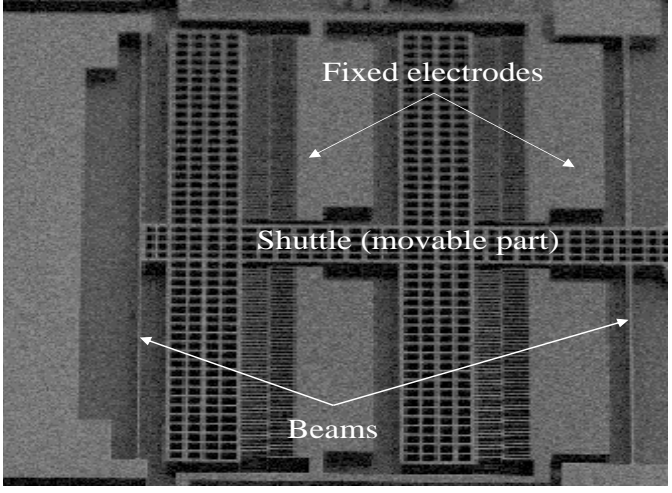

Fig. 6. Structure of ELCA after fabrication

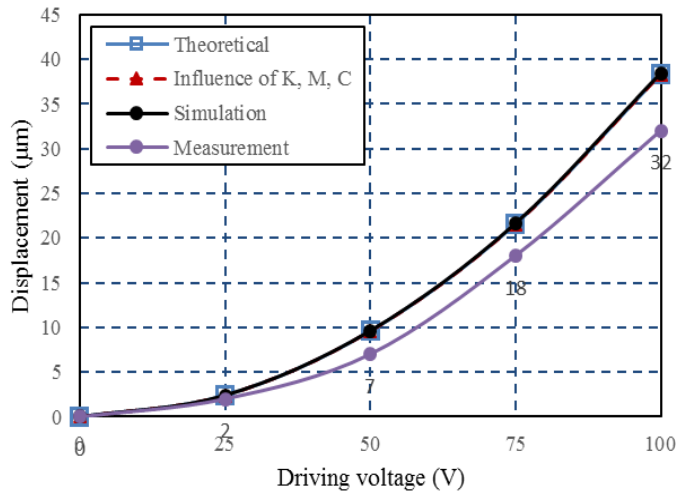

Fig. 7. Relation between the ELCA displacement and the driving voltage

conversion coefficients $(K, M, C)$ and confirms that at low frequencies (a few $\mathrm{Hz}$ ), the influence of inertial mass and air damping to ELCA's displacement are not significant.

The displacement error between the theoretical and the simulation is about $0.21 \%$ (see Fig. 7). Tiny deviation can be explained because of the difference of stiffness value calculated separately by theoretical and simulation. The difference of theoretical, simulation and measurement results are $19.81 \%$ and $20.06 \%$ at driving voltage of $100 \mathrm{~V}$, respectively. The reason of larger deviation is because of the voltage loss in the contact points between the probe tip and the anchor surface, as well as because of the fabricated comb gap is bigger than the design gap $g_{0}$. In other words, the real electrostatic force loaded on the ELCA will be smaller than the value calculated by Eq. (1).
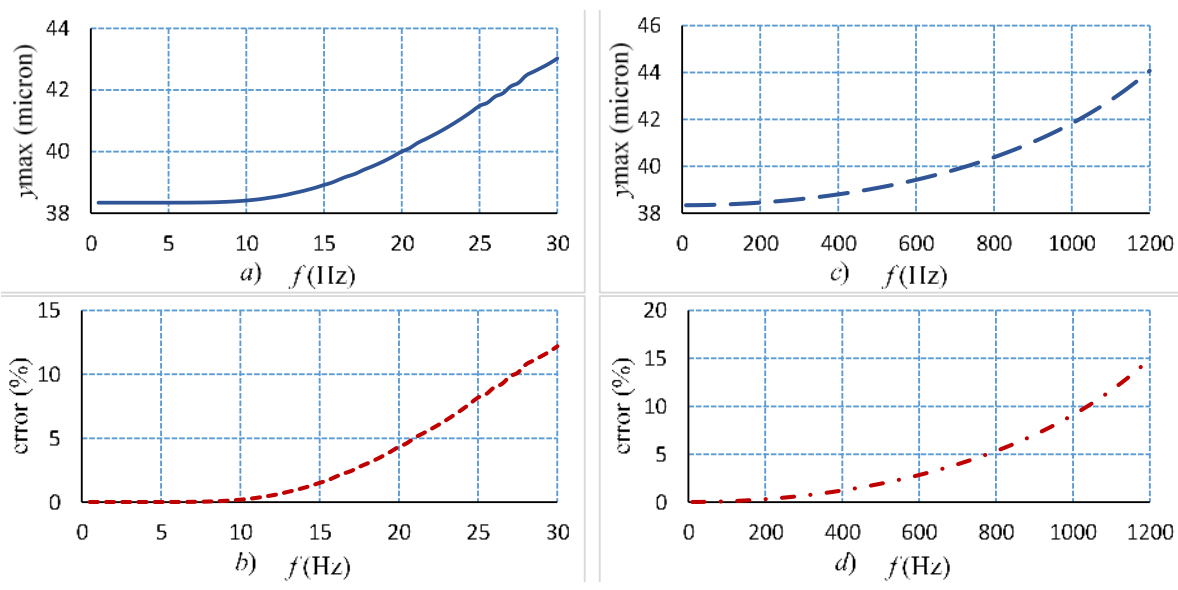

Fig. 8. Relation between maximum amplitude and the driving frequency

Fig. 8(a) shows that the maximum amplitude of the ELCA is nearly constant at low frequencies (less than $10 \mathrm{~Hz}$ ) in case of applying square wave voltage. The relative error of amplitude is $10 \%$ matching to working frequency of $27 \mathrm{~Hz}$ (Fig. 8(b)). In case of 
applying sine wave is shown in Fig. 8(c), the maximum amplitude is nearly consistently in a larger range of driving frequency; here, the error is also under $10 \%$ until ranging frequency up to $1 \mathrm{kHz}$ (Fig. 8(d)). Consequently, for the displacement of comb-drive structure, the sine wave is better than the square wave driving voltage with the range of working frequency is also larger.

The quality factor ( $Q$ factor) of the ELCA system can be calculated by [16]

$$
Q=\frac{\sqrt{K M}}{C} \text {. }
$$

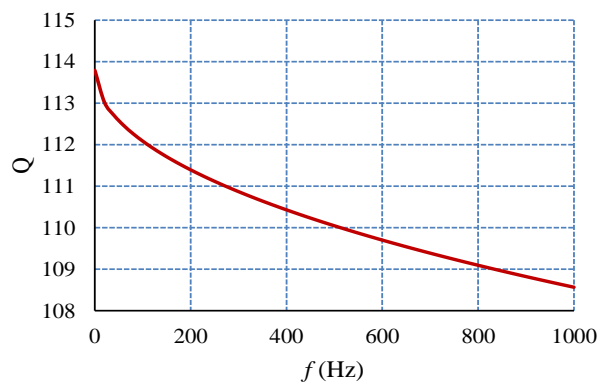

Fig. 9. $Q$ factor depends on a sine wave frequency

When frequency changing from $1 \mathrm{~Hz}$ to $1000 \mathrm{~Hz}, Q$ factor value decreases about $4.6 \%$ from 114 to under 109 (see Fig. 9). It shows that the $Q$ factor of ELCA reduces insignificantly, even thought the larger range of sine wave frequency value.

\section{CONCLUSION}

This paper studied and simulated the influence of vibration at different frequencies on the accuracy of an ELCA's displacement. Theoretical, simulation results and measurement data were compared and evaluated. Comments can be summarized as follows:

- The conversion coefficients $K, M$, and $C$ of the ELCA's differential motion equations are more exactly determined via the equivalent kinetic energy and air drag methods.

- In medium of air, while driving frequency is lower than $10 \mathrm{~Hz}$, the displacement of ELCA is nearly independent on damping and inertial force of the system.

- The displacement amplitude deviation in term of using sine wave voltage is smaller than $10 \%$ with driving frequency up to $1 \mathrm{kHz}$, while the displacement amplitude deviation if using square wave is approximately to $10 \%$ with driving frequency ranging is only $27 \mathrm{~Hz}$. It is clear that the sine wave frequency gives the displacement amplitude and working frequency range better than the square wave. This is a valuable suggestion for analysis and design of micro devices using the electrostatic comb-drive actuators such as micro motor, micro gripper or capacitive accelerometer.

\section{REFERENCES}

[1] W. C. Tang, T.-C. H. Nguyen, M. W. Judy, and R. T. Howe. Electrostatic-comb drive of lateral polysilicon resonators. Sensors and Actuators A: Physical, 21, (1-3), (1990), pp. 328-331. https://doi.org/10.1016/0924-4247(90)85065-c. 
[2] J. Bernstein, S. Cho, A. King, A. Kourepenis, P. Maciel, and M. Weinberg. A micromachined comb-drive tuning fork rate gyroscope. In Proceedings IEEE Micro Electro Mechanical Systems Workshop (MEMS'93). IEEE, (1993), pp. 143-148. https://doi.org/10.1109/MEMSYS.1993.296932.

[3] D. V. Dao, P. H. Pham, and S. Sugiyama. Multimodule micro transportation system based on electrostatic comb-drive actuator and ratchet mechanism. Journal of Microelectromechanical Systems, 20, (1), (2011), pp. 140-149. https://doi.org/10.1109/jmems.2010.2090503.

[4] P. H. Pham, L. B. Dang, V. H. Nguyen, and V. T. Dau. Development of new electrostatic micro cam system driven by elastic wings. Microsystem Technologies, 23, (12), (2017), pp. 5669-5675. https://doi.org/10.1007/s00542-017-3422-9.

[5] F. Khan, S. A. Bazaz, and M. Sohail. Design, implementation and testing of electrostatic SOI MUMPs based microgripper. Microsystem Technologies, 16, (11), (2010), pp. 1957-1965. https://doi.org/10.1007/s00542-010-1129-2.

[6] R. Legtenberg, A. W. Groeneveld, and M. Elwenspoek. Comb-drive actuators for large displacements. Journal of Micromechanics and Microengineering, 6, (3), (1996), pp. 320-329. https://doi.org/10.1088/0960-1317/6/3/004.

[7] S. Gupta, T. Pahwa, R. Narwal, B. Prasad, and D. Kumar. Optimizing the performance of MEMS electrostatic comb drive actuator with different flexure springs. In Proceedings of COMSOL Conference, Bangalore, India, (2012). pp. 1-6, https:/ / www.comsol.co.in/paper/download/152681/gupta_paper.pdf.

[8] M. Imboden, J. Morrison, E. Lowell, H. Han, and D. J. Bishop. Controlling levitation and enhancing displacement in electrostatic comb drives of MEMS actuators. Journal of Microelectromechanical Systems, 23, (5), (2014), pp. 1063-1072. https://doi.org/10.1109/jmems.2014.2303898.

[9] B. Borovic, F. L. Lewis, A. Q. Liu, E. S. Kolesar, and D. Popa. The lateral instability problem in electrostatic comb drive actuators: modeling and feedback control. Journal of Micromechanics and Microengineering, 16, (7), (2006), p. 1233. https://doi.org/10.1088/0960-1317/16/7/017.

[10] P. H. Pham and L. B. Dang. Influence of the side etching effect in DRIE on performance of electrostatic linear comb-drive actuators. Microsystem Technologies, 24, (5), (2018), pp. 22152222. https://doi.org/10.1007/s00542-017-3685-1.

[11] A. R. Kalaiarasi and S. H. Thilagar. Design and modeling of electrostatically actuated microgripper. In Proceedings of IEEE/ASME International Conference on Mechatronics and Embedded Systems and Applications (MESA). IEEE, (2012), pp. 7-11. https://doi.org/10.1109/mesa.2012.6275528.

[12] P. H. Pham, D. V. Dao, and S. Sugiyama. A micro transportation system (MTS) with large movement of containers driven by electrostatic comb-drive actuators. Journal of Micromechanics and Microengineering, 17, (10), (2007), pp. 2125-2131. https://doi.org/10.1088/0960$1317 / 17 / 10 / 026$.

[13] W. C. Tang. Electrostatic comb drive for resonant sensor and actuator applications. PhD thesis, University of California at Berkeley, USA, (1990).

[14] Y. Guan, S. Gao, H. Liu, and S. Niu. Acceleration sensitivity of tuning fork gyroscopes: Theoretical model, simulation and experimental verification. Microsystem Technologies, 21, (6), (2015), pp. 1313-1323. https://doi.org/10.1007/s00542-014-2185-9.

[15] M. H. Bao. Analysis and design principles of MEMS devices. Elsevier, Amsterdam, The Netherlands, 1st edition, (2005).

[16] S. S. Rao. Mechanical vibrations. Addison-Wesley, Boston, USA, 3rd edition, (1995). 